\title{
Organisation spatiale du peuplement de poissons dans le Bandama
}

\author{
Rose Danielle Bénié ABOUA *, Gervais N'ZI KONAN, Paul Essetchi KOUAMELAN, \\ Siaka BERTE et Mamadou BAMBA
}

Laboratoire d'Hydrobiologie, UFR Biosciences, Université de Cocody, 22 BP 582 Abidjan 22, Côte d'Ivoire. *Auteur correspondant, E-mail : abouabrd@yahoo.fr

\section{RESUME}

L'évolution des peuplements de poissons sur le Bandama a été étudiée en considérant quatre zones d'échantillonnage : en amont du lac de Kossou, dans les lacs de Kossou et de Taabo, entre les lacs de Kossou et de Taabo, et en aval du lac de Taabo. Au total, 74 espèces de poisson réparties en 49 genres, 28 familles et 10 ordres sont capturées, incluant un hybride (Tilapia hybride), deux espèces introduites (Heterotis niloticus et Oreochromis niloticus) et 13 espèces estuariennes/marines. Nous avons observé une augmentation de la richesse spécifique dans les milieux fluviatiles (amont de Kossou $=47$; entre les lacs $=51$; aval de Taabo = 54) par contre, cette richesse est faible dans les milieux lacustres (lac de Kossou $=37$ et lac de Taabo $=36$ ). La distribution de l'ichtyofaune en fonction des variables environnementales par l'analyse de ReDondance (RDA), indique que 14 variables : la transparence, la canopée, le $\mathrm{pH}$, la profondeur, la température, le taux de solides dissous, la conductivité, l'oxygène dissous la largeur, les bois morts, feuilles et racines, les plantes aquatiques, le mélange sable-gravier, la vitesse du courant et les roches influencent la répartition des poissons sur ce cours d'eau.

(C) 2010 International Formulae Group. All rights reserved.

Mots clés: Inventaire, Distribution, Ichtyofaune, Fleuve Bandama, Côte d'Ivoire.

\section{INTRODUCTION}

Des cours d'eau ivoiriens, le bassin du Bandama, et en particulier le cours principal du fleuve Bandama, est l'un des rares milieux aquatiques à avoir bénéficié de nombreuses études ichtyologiques (Daget et Iltis, 1965 ; Daget et al., 1973 ; Planquette et Lemasson, 1975 ; Mérona et al., 1978; Paugy et Lévêque, 1977 ; Mérona, 1981; Teugels et al., 1988) suite au programme de dix ans de surveillance écologique des milieux aquatiques initié par l'Orstom en 1974 en convention avec l'O.M.S. dans le cadre de la lutte contre l'onchocercose. Par ailleurs, l'aménagement de la vallée du Bandama (A.V.B.) qui a mis en place l'aménagement du barrage hydroélectrique de Kossou en 1972, suivi de celui de Taabo en 1980 ont été le point de départ d'un processus de développement économique et social de la région centre Bandama. En plus de cela, on dénombre une centaine de barrages hydroagricoles et agropastoraux dans la partie nord du cours d'eau (Traoré, 1996). Cependant, les barrages hydroélectriques ont fait l'objet d'étude d'impact et parfois d'âpres critiques sur leurs effets environnementaux (Gourène et al., 1999 ; Diarra et Soumaguel, 1997); de même que l'épandage d'insecticides dans les cours d'eau. Ainsi, le présent travail se propose d'étudier la composition des peuplements piscicoles dans 
les différentes parties du cours principal du fleuve Bandama : en amont du lac de Kossou, dans le lac de Kossou, entre les lacs Kossou et Taabo, dans le lac de Taabo et en aval du lac de Taabo vingt ans après les dernières études effectuées. Aussi, cette étude voudrait mettre en relief les relations entre les peuplements de poissons et les variables environnementales.

\section{MATERIEL ET METHODES}

Milieu d'étude et description des sites

Le cours principal du fleuve Bandama, lit principal du Bassin du Bandama est long de $1050 \mathrm{~km}$ pour un bassin versant de 97500 $\mathrm{km}^{2}$. Il prend sa source au Nord de la Côte d'Ivoire à $480 \mathrm{~m}$ d'altitude entre les villes de Korhogo et de Boundiali. En raison de son orientation Nord-Sud, il recouvre des zones différentes sur le plan climatique et biogéographique. Ses principaux affluents sont la Marahoué sur la droite et le N'Zi à gauche (Figure 1). Dans le cadre d'un processus de développement du pays, des barrages hydroélectriques, agropastoraux et hydroagricoles y ont été construits au centre et au nord (Lévêque et al., 1983 ; Traoré, 1996) créant des lacs artificiels.

Suivant le gradient amont-aval, cinq zones d'échantillonnage (Figure 1) ont été considérées :

En amont du lac de Kossou, on a un milieu formé de petites vasques, de radiers et de méandres. Cette zone renferme les stations B1, B2, B3, B4, B5, B6 et B7 ;

Dans les lacs de Kossou et de Taabo, on a un milieu lentique avec les stations B8, B9, B10, B11, B12, B13 et B14 pour le premier et les stations $\mathrm{B} 25$ et $\mathrm{B} 26$ pour le second;

Entre les lacs de Kossou et de Taabo, le niveau de l'eau est influencé par l'ouverture et la fermeture des vannes des barrages de Kossou et de Taabo, et reçoit en plus les eaux Marahoué. Ces différents éléments en font un hydrosystème particulier. Cette zone comprend les stations B15, B16, B17, B18, B19, B20, B21, B22, B23 et B24 ;
Et en aval du lac de Taabo, les stations (B27, B28, B29, B30, B31, B32, B33, B34, B35 et B36) sont caractérisées par la pénétration d'eaux estuariennes.

\section{Plan d'échantillonnage}

Les poissons ont été récoltés entre Octobre 2008 et Août 2009. Une batterie de 19 filets maillants monofilaments $(8,10,12$, $14,15,18,20,25,30,35,40,45,50,55,60$, $65,70,75$ et $80 \mathrm{~mm}$ de maille de vide) de 30 $\mathrm{m}$ de long et d'une hauteur de chute de $1,5 \mathrm{~m}$. Dans chaque station, il a été effectué deux pêches nocturnes $(17 \mathrm{~h}-7 \mathrm{~h})$ et une pêche diurne $(7 \mathrm{~h}-13 \mathrm{~h})$. En plus des filets maillants, un appareil de pêche à l'électricité de model "BACK PACK 12" a été utilisé dans les petits bras de faible profondeur $(<0,5 \mathrm{~m})$ aux stations B7, B23, B24, B33, B34, B35 et B36. Les poissons capturés ont été identifiés à l'aide de clés d'identification de Paugy et al. (2003a, 2003b), puis mesurés et pesés respectivement au millimètre et au gramme près. Au total, 29 stations de pêche au filet et 7 stations de pêche à l'électricité ont été étudiées.

\section{Variables environnementales}

La conductivité $(\mu \mathrm{S} / \mathrm{cm})$, le taux de solides dissous ou TDS (mg/l), l'oxygène $\left(\mathrm{mg} / \mathrm{l}\right.$ et en \%), le $\mathrm{pH}$, la température $\left({ }^{\circ} \mathrm{C}\right)$ et la transparence $(\mathrm{cm})$ ont été mesurés dans chaque station d'échantillonnage (Yao et al., 2005; Kouamé et al., 2008). Ils ont été mesurés respectivement avec un conductimètre CON 400/410, un oxymètre DO 330, un pH-mètre pH 300/310 donnant également la valeur de la température grâce à une sonde adaptée et un disque de secchi. Les variables de l'habitat notées sont: la largeur (m), la profondeur (m), la vitesse du courant (en chronométrant le déplacement d'une bouteille plastique de 0,5 1 à moitié remplie d'eau sur une distance donnée (McMahon et al., 1996)). Le taux de fermeture de la canopée, la couverture par les plantes aquatiques et le substrat ont été estimés et exprimés en pourcentage. 
Traitement des données et analyse statistique

Etant donné qu'aucune unité d'effort satisfaisante n'a pu être définie avec l'appareil de pêche électrique tant l'efficacité de ce genre d'engin est dépendante du milieu et de l'utilisateur (Mérona, 1981) et aussi du nombre de sites échantillonnés avec cet appareil, les comparaisons quantitatives dans les différents travaux ne seront pas prises en compte. Les résultats seront utilisés pour l'inventaire qualitatif et les comparaisons quantitatives ne vont tenir compte que des poissons capturés aux filets maillants.

L'analyse de redondance (RDA) est une méthode qui permet une interprétation des ordinations par une analyse simultanée des variables biotiques et abiotiques. Des séries de RDA ont été réalisées à partir d'une DCA (analyse des correspondances «détendancées ») dont la longueur du gradient en unité de déviation standard (SD) n'excède pas 4 SD. La sélection des variables environnementales qui expriment le mieux la distribution des poissons a été effectuée avec le test de permutation de Monte Carlo (permutation 499). L'analyse a été réalisée à partir des abondances relatives des espèces présentant un pourcentage supérieur à $1 \%$ de l'abondance totale dans chaque zone. Ces données ont subit une transformation logarithmique $[\log (\mathrm{x}+1)]$. Le logiciel statistique utilisé est le CANOCO (Ordination Canonique des Communautés, version 4.5) (Ter Braak et Smilauer, 2002).

\section{RESULTATS}

\section{Composition spécifique}

La composition de l'ichtyofaune recensée durant notre étude sur le cours principal du fleuve Bandama est présentée dans le Tableau 1. On dénombre 74 espèces de poissons reparties en 49 genres, 28 familles et 10 ordres. Cette répartition comprend 58 espèces d'eau douce, 13 espèces marines et/ou estuariennes (Elops lacerta, Pellonula leonensis, Caranx hippos, Trachinotus teraia, Gerres melanopterus, Sarotherodon galilaeus,
Sarotherodon melanotheron, Tilapia mariae, Tylochromis jentinki, Liza falcipinnis, Polydactylus quadrifilis, Awaous lateristriga et Eleotris vittata), deux espèces introduites (Heterotis niloticus et Oreochromis niloticus) et un hybride (Tilapia guineensis x Tilapia zillii). Les Characiformes ( $11 \%$ des familles et $12 \%$ des espèces) représentent $40 \%$ du nombre total de poissons capturés dans le fleuve. Ils sont suivis par l'ordre des Siluriformes (22\% des familles et $20 \%$ des espèces), des Osteoglossiformes (11\% des familles et $15 \%$ des espèces), des Cypriniformes (4\% des familles et $12 \%$ des espèces) et des Perciformes (37\% des familles et $35 \%$ des espèces) qui constituent respectivement $15 \%, 13 \%$ et $12 \%$ des captures. Et enfin, les clupeiformes (4\% des familles et $2 \%$ des espèces) qui totalisent $7 \%$ des poissons prélevés. Quant aux Polypteriformes, Elopiformes et Synbranchiformes (4\% des familles et $2 \%$ des espèces), leur abondance dans les effectifs représentent $1 \%$. Signalons aussi que des poissons appartenant à la famille des Soleidae (3 individus), Syngnatidae (3 individus) et Cyprinodontidae (un individu) n'ont pu être spécifiés; et que les espèces: Epiplatys chaperi, E. etzeli, Amphilius atesuensis, Barbus trispilos, B. macinensis, Micralestes occidentalis, Nannocharax fasciatus, Noelebias unifasciatus et les individus de la famille des Syngnatidae ont été récoltés avec l'appareil de pêche à l'électricité.

\section{Distribution de la richesse spécifique}

La distribution des poissons dans les zones d'échantillonnage considérées sur notre cours d'eau, est également indiquée au Tableau 1. Au vu de ces résultats, il ressort que 20 espèces de poissons sont communes aux différentes zones. Les espèces Barbus macinensis, Micralestes occidentalis, Nannocharax fasciatus, Noelebias unifasciatus capturées en amont du lac de Kossou, ne sont pas présentes dans les autres milieux. Entre Kossou et Taabo, les espèces Epiplatys etzeli, Amphilius atesuensis, Barbus 
trispilos et Marcusenius furcidens et Labeo sp ont été rencontrées dans cette zone. A Taabo, à l'exception des Barbus sp, toutes les autres espèces sont présentes ailleurs. En aval du lac de Taabo, aux espèces estuariennes et/ou marines (Elops lacerta, Caranx hippos, Trachinotus teraia, Gerres melanopterus, Tylochromis jentinki, Liza falcipinnis, Polydactylus quadrifilis, Awaous lateristriga et Eleotris vittata), s'ajoutent Marcusenius sp, Tilapia sp, et Thysochromis ansorgii qui sont seulement capturées dans cette partie du cours d'eau. Tandis qu'à Kossou, toutes les espèces récoltées sont présentes aux autres endroits. L'étude de la composition des peuplements ichtyologiques de ce fleuve (Figure 2) a montré que dans les milieux courants, le peuplement est dominé par la famille des Alestidae. Il est en grande partie représentée par les espèces: Brycinus longipinnis, $B$. imberi, Petrocephalus bovei, Pellonulla leonensis Schilbe intermedius, $S$. mandibularis, Raiamas senegalensis, en amont du lac de barrage de Kossou; par Brycinus longipinnis, B. imberi, $B$. macrolepidotus, Petrocephalus bovei, Schilbe mandibularis et Hemichromis fasciatus, entre les lacs de Kossou et de Taabo; et par Brycinus longipinnis en aval du lac de barrage de Taabo. Quant aux milieux lacustres, le peuplement est dominé par la famille des Cyprinidae (genre Barbus), des Cichlidae (Hemichromis fasciatus, H. bimaculatus) et des Mormyridae (Petrocephalus bovei) dans des proportions différentes.

Relation entre richesse spécifique et variables environnementales

Les ordinations obtenues par le logarithme des abondances des poissons et des variables du milieu dans les différentes zones considérées (les zones lacustres ont été analysées ensemble) sont présentées à la figure 3. Le test de Monte Carlo a permis de sélectionner les variables environnementales qui expriment mieux les corrélations espècesvariables.
Dans la partie fluviatile du Bandama, en amont du lac de Kossou, les variables environnementales qui influencent la distribution des espèces de poissons sont: le taux de solides dissous (TDS), la conductivité (Cnd), la température de l'eau (Te), les roches (R), la vitesse du courant (v), l'oxygène dissous $\left(\mathrm{O}_{2} \mathrm{mg} / \mathrm{l}\right)$ et la profondeur (Prof). Les variables Cnd, TDS et Te sont positivement corrélées aux axes I et II ; à ces variables sont associées les espèces Raiamas senegalensis (Rse) et Barbus ablabes (Babl). Les roches (R) sont positivement corrélées à l'axe II. Cette variable est liée à la distribution des espèces : Schilbe mandibularis (Sma), Tilapia zillii (Tzi) et Brycinus macrolepidotus (Bmal). Quant aux variables v, $\mathrm{O}_{2} \mathrm{mg} / \mathrm{l}$ et Prof, elles sont négativement corrélées aux axes I et II. La vitesse est associée aux espèces Synodontis schall (Ssc) et Brycinus imberi (Bim) et l'oxygène dissous, aux espèces Schilbe intermedius (Sin) et Marcusenius senegalensis (Mse).

Dans la partie lacustre, les paramètres environnementaux qui influencent la distribution des poissons sont : la transparence (Transp), l'oxygène dissous $\left(\mathrm{O}_{2} \mathrm{mg} / \mathrm{l}\right)$, la température de l'eau (Te), le taux de solides dissous (TDS), la conductivité (Cnd) et les plantes aquatiques (Paq). Les paramètres Paq, Cnd, TDS, Te, sont positivement corrélés à l'axe I. Les plantes aquatiques influencent la distribution Hemichromis fasciatus ( $\mathrm{Hfa}$ ) et Petrocephalus bovei (Pbo) alors que la conductivité présente une importante corrélation avec les espèces Chrysichthys maurus (Cma), Barbus sp (Bsp) et Schilbe mandibularis (Sma). Les espèces $H$. bimaculatus (Hbi), Brycinus longipinnis (Blo), Parailia pellucida (Ppe) sont associées au TDS alors que Pollimyrus isidori (Pis) est influencé par la température. Négativement corrélés aux axes I et II, les variables Transp et $\mathrm{O}_{2} \mathrm{mg} / \mathrm{l}$.

Dans la partie fluviatile qui se trouve entre les lacs de Kossou et de Taabo, ce sont le $\mathrm{pH}$, les plantes aquatiques (Paq), la température de l'eau (Te), la profondeur 
(prof), l'oxygène dissous $\left(\mathrm{O}_{2} \mathrm{mg} / \mathrm{l}\right)$ et le taux de fermeture de la canopée $(\mathrm{Fc})$ qui expriment le mieux la distribution des espèces. Les espèces $B$. longipinnis et $S$. mandibularis associées aux variables $\mathrm{O}_{2} \mathrm{mg} / \mathrm{l}$ et $\mathrm{Fc}$ sont corrélées à l'axe I. les variables Te et Prof sont négativement corrélées aux axes I et II. Les espèces Marcusenius ussheri (Mus), Hydrocynus forskalii (Hfo), Chromidotilapia guntheri (Cgu), Distichodus rostratus (Dro) y sont associées. Quant aux espèces $P$. bovei et B. imberi, on remarque qu'elles sont fortement corrélées au $\mathrm{pH}$ et aux plantes aquatiques (Paq),

Dans la partie fluviatile, en aval du lac de Taabo, les variables sélectionnées par le test de Monte Carlo sont la largeur (Larg), la transparence (Transp), les bois morts, feuilles et racines (BmFR), le taux de fermeture de la canopée ( $\mathrm{Fc}$ ), le $\mathrm{pH}$, la profondeur (Prof) et le mélange sable-gravier (SG). La largeur et la transparence positivement corrélées aux axes I et II influencent respectivement la distribution des espèces Petrocephalus bovei (Pbo), Chrysichthys nigrodigitatus (Cni), et Marcusenius ussheri (Mus), Brycinus imberi (Bim), B. longipinnis (Blo). Les variables $\mathrm{BmFR}$ et $\mathrm{Fc}$ positivement corrélées à l'axe I sont associées aux espèces, Hemichromis fasciatus (Hfa) et Schilbe mandibularis (Sma). Quant aux variables $\mathrm{pH}, \mathrm{SG}$ et Prof négativement corrélées aux axes I et II, elles ne sont associées à aucune espèce.

Tableau 1 : Liste des espèces de poissons recensées le long du fleuve Bandama.

\begin{tabular}{|c|c|c|c|c|c|c|c|}
\hline Familles & Espèces & Codes & $\begin{array}{l}\text { Amont } \\
\text { de } \\
\text { Kossou }\end{array}$ & $\begin{array}{c}\text { lac de } \\
\text { Kossou }\end{array}$ & $\begin{array}{c}\text { entre les } \\
\text { lacs de } \\
\text { Kossou } \\
\text { et } \\
\text { Taabo }\end{array}$ & $\begin{array}{l}\text { lac de } \\
\text { Taabo }\end{array}$ & $\begin{array}{c}\text { Aval } \\
\text { de } \\
\text { Taabo }\end{array}$ \\
\hline Polypteridae & Polypterus endlicheri & Pen & + & & + & & \\
\hline Elopidae & Elops lacerta $^{1}$ & Ela & & & & & + \\
\hline Osteoglossidae & Heterotis niloticus $^{2}$ & Hni & + & + & + & + & \\
\hline Notopteridae & Papyrocranus afer & Paf & & + & + & & + \\
\hline Clupeidae & Pellonula leonensis ${ }^{1}$ & Ple & + & + & + & + & + \\
\hline \multirow[t]{8}{*}{ Mormyridae } & Marcusenius furcidens & Mfu & & & + & & \\
\hline & Marcusenius senegalensis & Mse & + & + & + & + & + \\
\hline & Marcusenius ussheri & Mus & + & + & + & + & + \\
\hline & Marcusenius sp & Msp & & & & & + \\
\hline & Mormyrops anguilloides & Man & + & + & + & & + \\
\hline & Mormyrus rume & Mru & + & & + & & + \\
\hline & Pollimyrus isidori & Pis & + & + & & + & + \\
\hline & Petrocephalus bovei & Pbo & + & + & + & + & + \\
\hline Hepsetidae & Hepsetus odoe & Hod & + & & + & & + \\
\hline \multirow[t]{5}{*}{ Alestidae } & Alestes baremoze & Aba & + & & + & & + \\
\hline & Brycinus imberi & Bim & + & + & + & & + \\
\hline & Brycinus longipinnis & Blo & + & + & + & + & + \\
\hline & Brycinus macrolepidotus & Bmal & + & + & + & + & + \\
\hline & Brycinus nurse & Bnu & & & + & & + \\
\hline
\end{tabular}


R. D. B. ABOUA et al. /Int. J. Biol. Chem. Sci. 4(5): 1480-1493, 2010

\begin{tabular}{|c|c|c|c|c|c|c|c|}
\hline & Hydrocynus forskalii & Hfo & + & & \multirow[t]{2}{*}{+} & \multirow[t]{2}{*}{+} & \multirow[t]{2}{*}{+} \\
\hline & Micralestes occidentalis* & Moc & + & & & & \\
\hline \multirow[t]{3}{*}{ Distichodontidae } & Distichodus rostratus & Dro & + & & + & + & + \\
\hline & Nannocharax fasciatus* & $\mathrm{Nfa}$ & + & & & & \\
\hline & Noelebies unifasciatus * & $\mathrm{Nu} \mathrm{n}$ & + & & & & \\
\hline \multirow[t]{10}{*}{ Cyprinidae } & Barbus ablabes & $\mathrm{Bab}$ & + & + & & + & \\
\hline & Barbus macrops & Bmac & + & + & + & + & + \\
\hline & Barbus macinensis* & $\mathrm{Bmn}$ & + & & & & \\
\hline & Barbus sublineatus & $\mathrm{Bsu}$ & + & + & & & \\
\hline & Barbus trispilos* & Btr & & & + & & \\
\hline & Barbus sp & Bsp & & & & + & \\
\hline & Labeo coubie & Lco & + & + & + & + & + \\
\hline & Labeo parvus & Lpa & + & & + & + & + \\
\hline & Labeo sp & Lsp & & & + & & \\
\hline & Raiamas senegalensis & Rse & + & & + & & \\
\hline \multirow[t]{3}{*}{ Claroteidae } & Auchenoglanis occidentalis & Aoc & + & + & + & + & + \\
\hline & $\begin{array}{l}\text { Chrysichthys maurus } \\
\text { Chrysichthys }\end{array}$ & $\mathrm{Cma}$ & & + & + & + & + \\
\hline & nigrodigitatus & Cni & + & + & + & + & + \\
\hline
\end{tabular}

$1=$ espèces estuariennes/marines ; $2=$ espèces introduites $; 3=$ hybride $; *=$ espèces capturées qu'à la pêche électrique.

Tableau 1 (suite et fin)

\begin{tabular}{|c|c|c|c|c|c|c|c|}
\hline Familles & Espèces & Codes & $\begin{array}{c}\text { Amont de } \\
\text { Kossou }\end{array}$ & $\begin{array}{c}\text { lac de } \\
\text { Kossou }\end{array}$ & $\begin{array}{l}\text { entre les } \\
\text { lacs de } \\
\text { Kossou } \\
\text { et Taabo }\end{array}$ & $\begin{array}{l}\text { lac de } \\
\text { Taabo }\end{array}$ & $\begin{array}{c}\text { Aval } \\
\text { de } \\
\text { Taabo }\end{array}$ \\
\hline Amphiliidae & Amphilius atesuensis* & Aat & & & + & & \\
\hline \multirow[t]{3}{*}{ Schilbeidae } & Parailia pellucida & Ppe & + & + & + & + & + \\
\hline & Schilbe intermedius & Sin & + & + & + & + & + \\
\hline & Schilbe mandibularis & Sma & + & + & + & + & + \\
\hline \multirow[t]{3}{*}{ Clariidae } & Clarias anguillaris & Can & + & + & + & + & + \\
\hline & Heterobranchus isopterus & His & + & & + & & + \\
\hline & Heterobranchus longifilis & Hlo & + & & & + & \\
\hline Malapteruridae & Malapterurus electricus & Mel & + & + & + & & + \\
\hline \multirow[t]{3}{*}{ Mochokidae } & Synodontis bastiani & Sba & + & + & + & + & + \\
\hline & Synodontis punctifer & $\mathrm{Spu}$ & + & + & + & + & + \\
\hline & Synodontis schall & Ssc & + & & + & + & + \\
\hline \multirow[t]{2}{*}{ Aplocheilidae } & Epiplatys chaperi* & Ech & & & + & & + \\
\hline & Epiplatys etzeli* & Eet & & & + & & \\
\hline Channidae & Parachanna obscura & Pob & & & + & + & + \\
\hline Centropomidae & Lates niloticus & Lni & + & + & + & & + \\
\hline \multirow[t]{2}{*}{ Carangidae } & Caranx hippos $^{1}$ & Chi & & & & & + \\
\hline & Trachinotus teraia ${ }^{1}$ & Tte & & & & & + \\
\hline Gerreidae & Gerres melanopterus $^{1}$ & Gme & & & & & + \\
\hline \multirow[t]{2}{*}{ Cichlidae } & $\begin{array}{l}\text { Chromidotilapia guntheri } \\
\text { Hemichromis }\end{array}$ & $\mathrm{Cgu}$ & + & + & + & + & + \\
\hline & bimaculatus & $\mathrm{Hbi}$ & + & + & + & + & + \\
\hline
\end{tabular}




\begin{tabular}{|c|c|c|c|c|c|c|c|}
\hline & Hemichromis fasciatus & Hfa & + & + & + & + & + \\
\hline & Oreochromis niloticus ${ }^{2}$ & Oni & + & + & & + & \\
\hline & Sarotherodon galilaeus ${ }^{1}$ & Sga & + & + & + & + & + \\
\hline & melanotheron ${ }^{1}$ & Sme & & + & + & & \\
\hline & Thysochromis ansorgii & Tan & & & & & + \\
\hline & Tilapia hybride ${ }^{3}$ & Thy & & + & + & + & + \\
\hline & Tilapia guineensis & Tgu & & + & & + & \\
\hline & Tilapia mariae ${ }^{1}$ & Tma & & + & + & & + \\
\hline & Tilapia zillii & Tzi & + & + & + & + & + \\
\hline & Tylochromis jentink $i^{1}$ & Tje & & & & & + \\
\hline & Tilapia $s p$ & Tsp & & & & & + \\
\hline Mugilidae & Liza falcipinnis $^{1}$ & Lfa & & & & & + \\
\hline Polynemidae & Polydactylus quadrifilis ${ }^{1}$ & Pqu & & & & & + \\
\hline Gobiidae & Awaous lateristriga 1 & Ala & & & & & + \\
\hline Eleotridae & Eleotris vittata $^{1}$ & Evi & & & & & + \\
\hline Anabantidae & Ctenopoma petherici & Cpe & + & + & + & + & + \\
\hline Mastacembelidae & $\begin{array}{l}\text { mastacembelus } \\
\text { nigromarginatus }\end{array}$ & Mni & + & & + & & \\
\hline 28 & 74 & & 47 & 37 & 51 & 36 & 54 \\
\hline
\end{tabular}

\section{DISCUSSION}

En se référant aux travaux dernièrement effectués sur le fleuve Bandama par Teugels et al. (1988), on note que plus d'espèces ont été récoltées dans leurs travaux (96 espèces), contrairement à la liste d'espèces présentée dans le tableau 1 (74 espèces). Comparativement à leurs données, il ressort que plusieurs espèces n'ont pas été capturées lors de nos échantillonnages aux filets maillants. Ces poissons sont pour la plupart de petits poissons $(12-67 \mathrm{~mm})$ capturés à la pêche électrique (Cyprinodontidae, Syngnatidae, des petits Barbus, Alestidae et Distichodontidae) et de 14 espèces capturées aux filets: Brienomyrus brachyistius, Mormyrus hasselquistii, Mormyrops breviceps, Laeviscutella dekimpei, Protopterus annectens, Labeo coubie, Raimas nigeriensis, Citharinus eburneensis, Kribia nana, Parasicydium bandamae, Nematogobius maindroni, Clarias ebriensis, C. buettikoferi, C. gariepinus). Cependant, certaines espèces sont rapportées pour la première fois. Il s'agit de Gerres melanopterus, Caranx hippos, Trachinotus teraia, Liza falcipinnis, Eleotris vittata et Epiplatys etzeli. Quant aux espèces Elops lacerta, Polydactylus quadrifilis et Thysochromis ansorgii non capturées par Teugels et al. (1988), elles ont été prises lors de nos campagnes d'échantillonnages, et ont déjà été mentionnées par Mérona (1981) pour les deux premières et par Daget et Iltis (1965) et Daget et al. (1973) pour la troisième espèce. Les différences observées au niveau des données précédentes et récentes de la faune piscicole du fleuve, pourraient provenir, d'après Kouamélan (2003) des méthodes de pêches utilisées, des types d'habitats échantillonnés et de la période d'échantillonnage. Mais quelle que soit la différence qualitative et quantitative de l'ichtyofaune de ce cours d'eau, on constate que les Perciformes, les Siluriformes, les Osteoglossiformes et les Characiformes sont les ordres les plus riches en familles et en espèces; et les familles qui dominent le peuplement sont les Cyprinidae, les Mormyridae, les Cichlidae et les Alestidae. Ces résultats ont également été observés sur d'autres bassins de la Côte d'Ivoire et de l'Afrique de l'Ouest (Teugels et al., 1988 ; Lévêque et al., 1990, 1992; Lévêque et Paugy, 1999 ; Da Costa et al., 2000 ; Konan et al., 2006). 


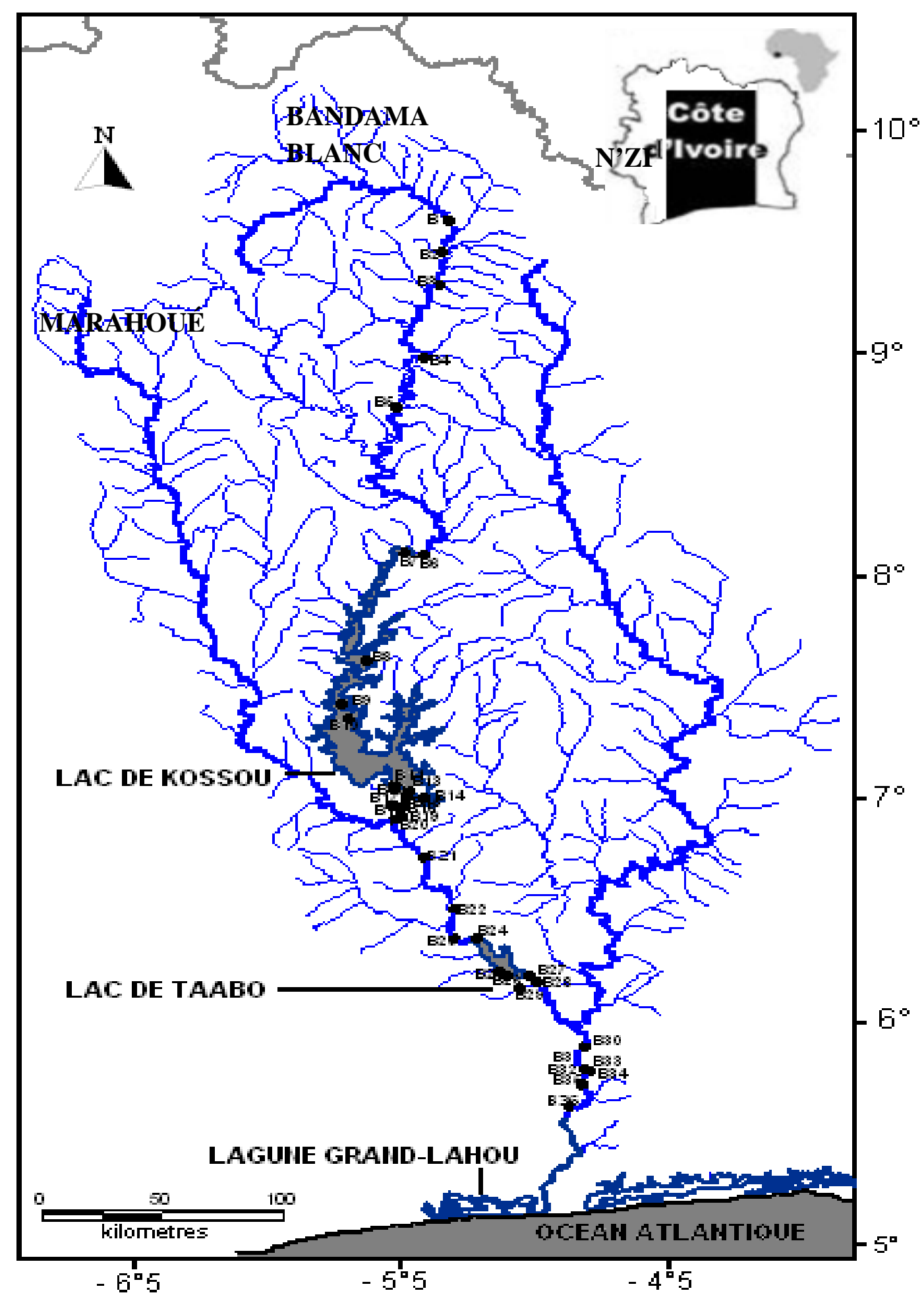

Figure 1 : Milieu d'étude et localisation des différentes stations d'échantillonnage.

- : Stations d'échantillonnage. 


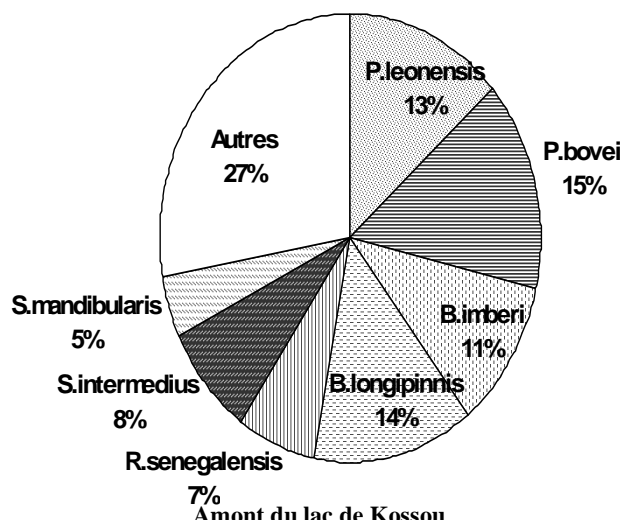

Amont du lac de Kossou

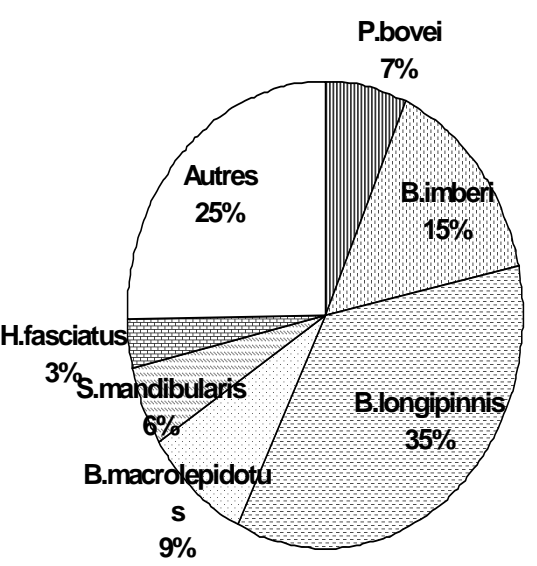

Entre les lacs de Kossou et de Taabo

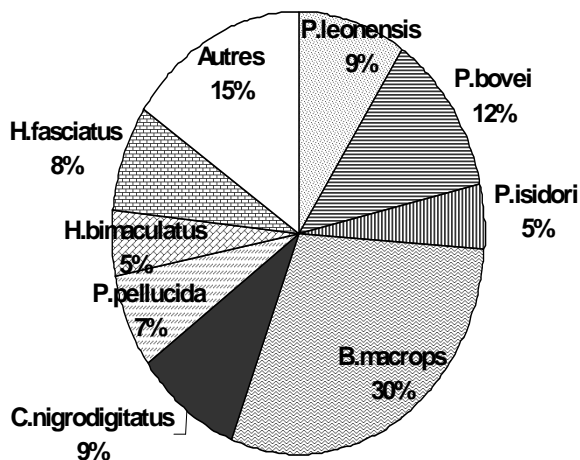

Lac de Kossou

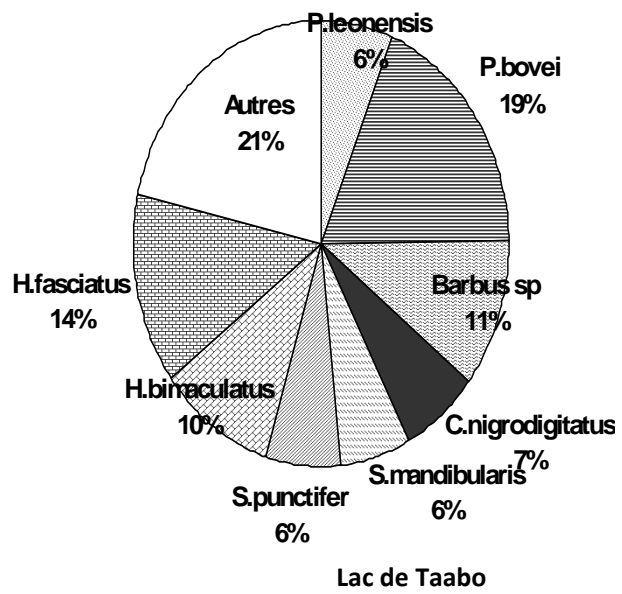

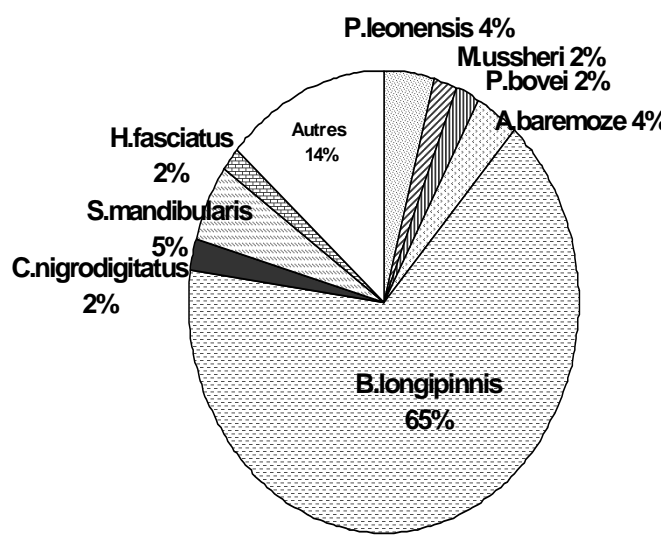

Aval du lac de Taabo

Figure 2 : Proportions numériques relatives des principales espèces de poissons capturés dans le Bandama blanc. 

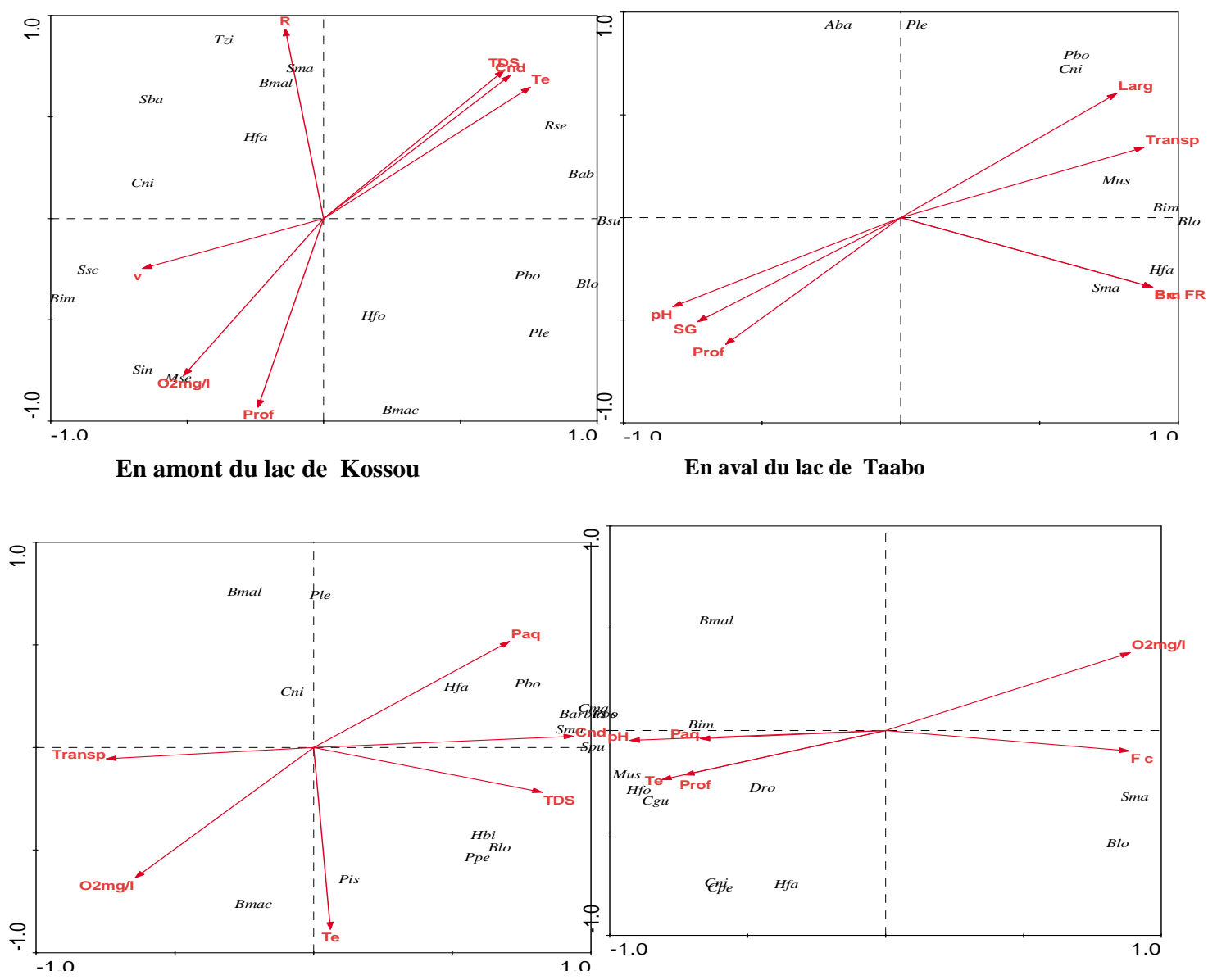

Dans les lacs de Kossou et de Taabo

Entre les lacs de Kossou et de Taabo

Figure 3 : Relations entre les espèces de poissons et les variables environnementales le long du Bandama blanc.

Transp=transparence, $\mathrm{Fc}=$ taux de fermeture de la canopée, Prof=profondeur, Te=température, TDS=taux de solides dissous, Cnd=conductivité, oxygène dissous $=\mathrm{O}_{2} \mathrm{mg} / \mathrm{l}, \mathrm{Paq}=$ plantes aquatiques, $\mathrm{Larg}=\mathrm{largeur}$, BmFR=bois morts, feuilles et racines, SG=mélange sable, Tzi=Tilapia zillii, Sba=Synodontis bastiani, Cni=Chrysichthys nigrodigitatus, Sma=Schilbe mandibularis, Bmal=Brycinus macrolepidotus, $\mathrm{Hfa}=$ Hemichromis fasciatus, $\mathrm{Ssc}=$ Synodontis schall, $\mathrm{Bim}=$ Brycinus imberi, $\mathrm{Sin}=$ Schilbe intermedius, Mse=Marcusenius senegalensis, $\mathrm{Rse}=$ Raiamas senegalensis, $\mathrm{Bab}=$ Barbus ablabes, $\mathrm{Bsu}=$ Barbus sublineatus, $\mathrm{Pbo}=$ Petrocephalus bovei, $\mathrm{Blo}=$ Brycinus longipinnis, $\mathrm{Pl}=$ Pellonula leonensis, $\mathrm{Hfo}=$ Hydrocynus forskalii, $\mathrm{Bmac}=$ Barbus macrops, $\mathrm{Aba}=$ Alestes baremoze, Mus=Marcusenius ussheri, $\mathrm{Spu}=$ Synodontis punctifer, $\mathrm{Hbi}=$ Hemichromis bimaculatus, $\mathrm{Pis}=$ Pollimyrus isidori, $\mathrm{Ppe}=$ Parailia pellucida, $\mathrm{Cma}=$ Chrysichthys maurus, $\mathrm{Cgu}=$ Chromidotilapia guntheri, Dro=Distichodus rostratus, $\mathrm{Cpe}=$ Ctenopoma petherici .

La distribution de l'ichtyofaune dans le fleuve Bandama blanc montre une augmentation de la richesse spécifique le long du gradient amont-aval, précisément dans les milieux courants (47 espèces en amont du lac de Kossou, 51 espèces entre les deux lacs et 54 espèces en aval du lac de Taabo). De l'aval du lac de Taabo à l'embouchure du fleuve Bandama, ont été recensées des espèces estuariennes/marines. Cette partie du fleuve correspondrait donc à la zone sous influence marine. Cependant, Lévêque et al. (1983) ont étendu cette zone de l'embouchure au confluent avec le N'Zi. Les zones faunistiques 
mises en évidence, à savoir l'amont du lac de Kossou, entre les lacs de Kossou et de Taabo et l'aval du lac de Taabo, montre en général une augmentation du nombre des espèces le long du gradient amont-aval par l'addition successive d'espèces plutôt que par un remplacement de celles-ci. Cette disposition obéit aux observations relatives à la zonation longitudinale des cours d'eau africains énoncé par Welcomme (1985). Des résultats similaires ont été obtenus par Kouamé et al. (2008) sur le fleuve Sassandra en Côte d'Ivoire. Toutefois, il a été observé dans les lacs de Kossou et de Taabo situé au centre du cours d'eau, des valeurs de richesse spécifique basses (lac Kossou, $\mathrm{n}=37$; lac de Taabo, $\mathrm{n}=$ 36) comparées à celles des milieux courants. Cette faible richesse pourrait être due à la construction des barrages de Kossou et Taabo sur le lit principal du Bandama blanc. En effet, la mise en place des barrages va entrainer des modifications profondes du milieu aquatique pouvant conduire à la disparition d'espèces éventuellement présentes dans ces zones et qui ne s'adapteraient pas aux nouvelles conditions lentiques, désoxygénées au fond créées par la mise en eau. Selon Kouassi (1979), cette évolution du peuplement ichtyologique répond au schéma habituellement observé pour les lacs de barrages africains avec la disparition et l'expansion de certaines espèces. Contrairement aux observations de Kouassi (1979) qui signale l'expansion d'autres espèces appartenant aux Alestidae $(A$. baremoze) et Centropomidae, les résultats de nos pêches dans les eaux des deux lacs montrent que les espèces Hemichromis fasciatus, Petrocephalus bovei et Barbus sp appartenant respectivement aux Cichlidae, Mormyridae et Cyprinidae dominent le peuplement. Fait également observé dans le lac d'Ayamé I par Da Costa et al. (2003). A ce sujet, Koné et al. (2003) indiquent que la création des lacs de barrage est souvent accompagnée de la colonisation des espèces de Cichlidae et de certaines espèces fluviatiles. Cet effet semble correspondre à ce qui a été souvent observé dans les barrages africains et asiatiques où les espèces pélagiques «explosent» (Welcomme, 1979). Dans ces milieux, les périodes de crues augmentent les surfaces immergées et la submersion de la végétation environnante entraine la mise à disposition d'une importante quantité de nutriments disponibles pour les poissons. L'étude de la composition ichtyologique des milieux courants sur le Bandama blanc a montré qu'une grande partie de ces cours d'eau présente un peuplement relativement homogène caractérisé par la dominance $B$. longipinnis, $B$. imberi et $B$. macrolepidotus. Un tel peuplement a déjà été signalé dans les fleuves tropicaux africains (Lévêque et al., 1983). La présence d'un peuplement homogène serait due au fait que la majeure partie des cours d'eau se déroule sur des zones de faible pente et que ce facteur ne joue pas un rôle aussi important que dans d'autres régions où des zonations beaucoup plus nettes ont été constatées. C'est le cas en Afrique de certains cours d'eau comme la rivière Luanga du bassin du Zaïre (Malaisse, 1976), des rivières d'Afrique du Sud (Harrisson, 1965). Les observations de terrain ont permis de constater que $B$. longipinnis présent sur toute la longueur du fleuve, est l'espèce la plus abondante. Mais elle parait plus abondante en aval du lac de barrage de Taabo (où elle représente $66 \%$ du nombre d'individus), entre les deux lacs (35\% de ceux-ci) et moins en amont du lac de barrage de Kossou (14\%) et dans les deux lacs ( $\geq 5 \%$ ). Les observations de terrain de Lévêque et al. (1983) ont également montré que cette espèce est abondante dans le cours supérieur et inférieur mais, absente du cours moyen, c'està-dire dans les lacs de Kossou et de Taabo et dans le cours d'eau entre ces lacs. L'abondance de cette espèce dans ces milieux (constitués d'une alternance de vasques et de radiers) serait due au fait qu'elle y trouve les conditions favorables à sa reproduction, à sa croissance.

A cette distribution des peuplements, correspondent plus ou moins des variables 
environnementales. Dans cette étude, 14 variables du milieu identifiées (en considérant toutes les zones d'échantillonnage) sont présentées comme celles qui influencent le plus la distribution des espèces de poissons. Certaines de ces variables : la transparence, le taux de fermeture de la canopée, le pH, la profondeur, la température de l'eau, le taux de solides dissous, la conductivité, l'oxygène dissous et les plantes aquatiques sont influentes dans deux zones d'échantillonnage au moins. Par contre, les variables comme la largeur, les bois morts, les feuilles et les racines et le mélange sable-gravier influencent la distribution des poissons en aval du lac de Taabo, alors que la vitesse et les roches influencent celle des poissons en amont du lac de Kossou. En Côte d'Ivoire, plusieurs études réalisées sur différents hydrosystèmes ont établi des relations entre espèces et variables environnementales (Da Costa et al., 2003 ; Kouamélan et al., 2003 ; Yao et al., 2005 ; Kouadio et al., 2006 ; Kouamé et al., 2008). Chacune de ces variables joue directement ou indirectement un rôle important sur la faune ichtyque. Au niveau de l'Agnébi et de la Bia, Da Costa et al. (2000) mentionnent qu'une amplitude faible de variation de la température moyenne de l'eau peut avoir une influence sur l'abondance et la répartition temporelle des espèces de poissons. Dans ces mêmes rivières, le TDS et la conductivité apparaissent comme les principaux facteurs discriminants. En Afrique de l'ouest, Hugueny (1989) a trouvé une relation entre le nombre d'espèce et la largeur dans la rivière Niadan en Guinée. L'importance de la vitesse du courant, de la profondeur de l'eau et du substrat dans la détermination de la composition du peuplement ichtyologique a aussi été relevée par Hugueny (1990). Dans la rivière N'tem au Cameroun, Kamdem Toham et Teugels (1998) ont trouvé que certaines espèces du genre Barbus avaient des préférences pour des taux d'oxygène élevés et pour une large couverture de la canopée. La végétation submergeant, quant à elle joue un rôle clé dans la détermination de la température, de la production primaire, de la physico-chimie, du régime hydrologique et de la distribution de la guilde trophique à l'intérieur des cours d'eau (Welcomme et Mérona, 1988). Les bois morts, feuilles, et racines et leurs débris influencent les caractéristiques de l'habitat tel que la profondeur, la transparence et le courant (Angermeier et Karr, 1984) tout en assurant aux poissons une source disponible de nourriture et une protection contre les prédateurs.

\section{Conclusion}

Sur le cours principal du fleuve Bandama, 74 espèces de poissons ont été récoltées. Le peuplement est réparti de façon homogène et présente des assemblages d'espèces caractéristiques. C'est ainsi qu'en amont du lac de Kossou, entre les lacs de Kossou et de Taabo et en aval du lac de Taabo, le peuplement est en majorité constitué de Brycinus longipinnis, B. imberi et de $B$. macrolepidotus. Par contre, dans les lacs de kossou et de taabo, ce sont les espèces Petrocephalus bovei, Hemichromis fasciatus et les petits Barbus qui dominent le peuplement. les facteurs environnementaux tels que la transparence, le taux de fermeture de la canopée, le $\mathrm{pH}$, la profondeur, la température de l'eau, le taux de solides dissous, la conductivité, l'oxygène dissous, les plantes aquatiques, la largeur, les bois morts, les feuilles et les racines et le mélange sablegravier et les facteurs anthropiques (les barrages hydroélectriques de Kossou et de Taabo) expliquent la distribution des espèces de poissons dans cet hydrosystème.

\section{REMERCIEMENTS}

Ce travail a été réalisé dans le cadre d'un Programme d'Appui Stratégique à la Recherche Scientifique (PASRES) sur le "Développement d'un indice d'intégrité biotique pour la préservation de la biodiversité du fleuve Bandama". Nous tenons à remercier toute l'équipe pour la collecte et le traitement des données. 


\section{REFERENCES}

Angermeier PL, Karr JR. 1984. Relationships between woody debris and fish habitat in a small warm water stream. Transactions of American Fisheries Society, 113: 716726.

Da Costa KS, Gourène G, Tito De Morais L, Thys Van Den Audenaerde DFE. 2000. Caractérisation des peuplements ichtyologiques de deux fleuves côtiers Ouest-Africains soumis à des aménagements hydroagricoles et hydroélectriques. Vie et Milieu, 50: 6577.

Daget J, Iltis A. 1965. Poissons de Côte d'Ivoire (eaux douces et saumâtres). Mém. I. F. A. N., 74: 385.

Daget J, Planquette N, Planquette P. 1973. Premières données sur la dynamique des peuplements de poissons du Bandama (Côte d'Ivoire). Bull. Mus. Nat. Hist. Nat., 151(3): 129-142.

Diarra A, Soumaguel A. 1997. Influence du barrage Sélingué sur le régime hydrologique du fleuve Niger. Sustainability of Water Resources under Increasing uncertainly (Proceedings of the habitat symposium SI). IAHS., 240: 277-286.

Gourène G, Teugels GG, Hugueny B, Thys Van den Audenaerde DFE. 1999. Evaluation de la diversité ichtyologique d'un basssin Ouest-Africain après la construction d'un barrage. Cybium, 23(2) : 147-160.

Harrisson AD. 1965. River zonation in Southern Africa. Arch. Hydrobiol., 61: 380-386.

Hugueny B. 1989. Biogéographie et structure des peuplements de poisson d'eau douce de l'Afrique de l'Ouest: approches quantitatives. Thèse Université de Paris VII, p. 295.

Hugueny B. 1990. Richesse des peuplements de poissons dans le Niandan (Haut Niger, Afrique) en fonction de la taille de la rivière et de la diversité du milieu. Revue d'Hydrobiologie Tropicale, 23: 351-364.
Kamdem Toham A, Teugels GG. 1998. Diversity patterns of fish assemblages in the Lower Ntem River Basin (Cameroon), with notes on potential effect of deforestation. Archiv. für Hydrobiologie, 141: 421-446.

Konan FK, Leprieur F, Ouattara A, Brosse S, Grenouillet G, Gourène G, Winterton P, Lek S. 2006. Spatio-temporal patterns of fish assemblages in coastal West African rivers: a self-organizing map approach. Aquat. Living Resour., 19: 361-370.

Koné T, Teugels GG, N'Douba V, Gooré Bi G, Kouamélan EP. 2003. Premières données sur l'inventaire et la distribution de l'ichtyofaune d'un petit bassin côtier ouest-africain: Rivière Gô (Côte d'Ivoire). Cybium, 27(2): 101-106.

Kouadio NF, Kouamélan EP, N'Douba V, Koné T, Snoeks J, Ollevier F. 2006. Update of fish biodiversity and impact of human activities on the community structure, Mé River (Ivory Coast). Journal of Biological Sciences, 6: 805814.

Kouamé KA, Yao SS, Gooré Bi G, Kouamélan EP, N'Douba V, Kouassi NJ. 2008. Influential environmental gradients and patterns of fish assemblages in a West African basin. Hydrobiologia, 603: 159-169.

Kouamélan EP, Teugels GG, N'Douba V, Gooré Bi G, Koné T. 2003. Fish diversity and its relationship with environment variables in West African basin. Hydrobiologia, 505: 139-146.

Kouassi N. 1979. Installation et évolution des populations piscicoles dans le lac de barrage de Kossou (Côte d'Ivoire) entre 1972 et 1977. Ann. Univ. Abidjan, E., 12: 159-174.

Lévêque C, Dejoux C, Iltis A. 1983. Limnologie du fleuve Bandama, Côte d'Ivoire. Hydrobiologia, 100: 113-141.

Lévêque C, Paugy D, Teugels GG. 1990. Faune des Poissons d'Eaux Douces et Saumâtres de l'Afrique de l'Ouest (Tome 
1). Editions ORSTOM (Paris) et MRAC (Tervuren) ; 1-384.

Lévêque C, Paugy D, Teugels GG. 1992. Faune des Poissons d'Eaux Douces et Saumâtres de l'Afrique de l'Ouest (Tome 2). Editions ORSTOM/MRAC ; 385-902.

Lévêque C, Paugy D. 1999. Impact des activités humaines. In Les Poissons des Eaux Continentales Africaines: Diversité, Ecologie, Utilisation par l'Homme, Lévêque C, Paugy D (eds). IRD: Paris ; 365-383.

McMahon TE, Zale AV, Orth DJ. 1996. Aquatic habitat measurements. In Fisheries Techniques, Murphy BR, Willis DW (eds). American Fisheries Society: Bethesda, Maryland, USA; 83-120.

Malaisse F. 1976. Ecologie de la rivière Luanga. In Exploration Hydrobiologique $d u$ Bassin du Lac Bangweolo et du Luapula, Symoem JJ (ed). 17, fasc. 2 : $151 \mathrm{p}$.

Mérona B. 1981. Zonation ichtyologique du bassin du Bandama. Revue Hydrobiol. Trop., 14: 63-75.

Mérona B, Lévêque C, Paugy D, Albaret JJ, Bigorne R, Herbinet P. 1978. Observations sur la Faune Ichtyologique du Bandama en Amont du Barrage de Kossou (Côte d'Ivoire). ORSTOM : Bouaké ; 193p.

Paugy D, Lévêque C. 1977. Observations sur la Faune Ichtyologique du Bandama en Aval du Barrage de Kossou (Côte d'Ivoire). ORSTOM : Bouaké; 63 p.

Paugy D, Lévêque C, Teugels GG. 2003a. Faune des Poissons d'Eaux Douces et Saumâtres de l'Afrique de l'Ouest. Editions IRD: Paris.
Paugy D, Lévêque C, Teugels GG. 2003b. Faune des Poissons d'Eaux Douces et Saumâtres de l'Afrique de l'Ouest. Editions IRD: Paris.

Planquette R, Lemasson J. 1975. Le peuplement de poissons du Bandama blanc en pays Baoulé. Ann. Univ. Abidjan E., 8: 77-121.

Ter Braak CJF, Smilauer P. 2002. CANOCO reference manual and Canodraw for Windows user's guide: software for canonical community ordination (version 4.5). Microcomputer Power, New York.

Teugels GG, Lévêque C, Paugy D, Traoré K. 1988. Etat des connaissances sur la faune ichtyologique des bassins côtiers de Côte d'Ivoire et de l'ouest du Ghana. Revue d'Hydrobiologie Tropicale, 21: 221-237.

Traoré K. 1996. Etat des connaissances sur les pêcheries continentales ivoiriennes. Rapport de consultation. PROJET FAO TCP/IVC/4553, IDESSA, Bouaké, Côte d'Ivoire.

Welcomme RL. 1979. Fisheries Ecology of Floodplain Rivers. Longman: London; $317 \mathrm{p}$.

Welcomme RL. 1985. Rivers fisheries. FAO Fish. Techn. Pap., 262: 330.

Welcomme RL, Mérona B. 1988. Fish communities of rivers. In Biology and Ecology of African Freshwater Fishes, Lévêque C, MN Bruton, GW Ssentongo (eds). Editions de l'ORSTOM: Paris; 251-276.

Yao SS, Kouamélan EP, Koné T, N'Douba V, Gooré Bi G, Ollevier F, Thys Van Den Audenaerde DFE. 2005. Fish communities along environment gradients within the Comoé River basin, Côte d'Ivoire. African Journal of Aquatic Science, 30: 195-204. 